\title{
Triboelectric Nanogenerators and Power-Boards from Cellulose Nanofibrils and Recycled Materials
}

\author{
Chunhua Yao ${ }^{\mathrm{a}},{ }^{\dagger}$ Alberto Hernandez ${ }^{\mathrm{a}},{ }^{\dagger}$ Yanhao $\mathrm{Yu}^{\mathrm{a}}$, Zhiyong Cai ${ }^{\mathrm{b},{ }^{*}}$ and Xudong Wang ${ }^{\mathrm{a}, *}$ \\ ${ }^{a}$ Department of Material Sciences and Engineering, \\ University of Wisconsin-Madison, Madison, WI 53706, USA \\ E-mail: xudong@engr.wisc.edu \\ ${ }^{\mathrm{b}}$ Forest Products Laboratory \\ USDA Forest Service, Madison, WI 53706, USA \\ E-mail: zcai@fs.fed.us \\ $\uparrow$ These two authors contribute equally
}

\begin{abstract}
This paper reports the implementation of renewable, biodegradable, and abundant cellulose nanofibrils (CNFs) in triboelectric nanogenerator (TENG) development. Flexible and transparent CNF thin films are triboelectric positive material with nanoscale surface roughness. They are paired with FEP (fluorinated ethylene propylene) to assemble TENG devices, which exhibit comparable performance to the reported TENG devices built on synthetic polymers. CNF-based TENG is further integrated within a fiberboard made from recycled cardboard fibers using a chemical-free cold pressing method. The fiberboard produces up to $\sim 30 \mathrm{~V}$ and $\sim 90 \mu \mathrm{A}$ electric outputs when subjected to a normal human step. This development shows great promises in creating large-scale and environmentally sustainable triboelectric board for flooring, packaging and supporting infrastructures from CNF and other natural wood-extracted materials.
\end{abstract}

Keywords: Triboelectric nanogenerator; Cellulose nanofibrils; Mechanical energy harvesting; Fiberboard; Recycled materials 


\section{Introduction}

Mechanical energy from the environment (e.g. ambient vibrations or activities of human body) ${ }^{[1,}$

${ }^{2]}$ represents a unique and sustainable energy source that perfectly suits for powering portable electronics and unattended devices. ${ }^{[2-4]}$ In 2012, the triboelectric nanogenerator (TENG) was first reported ${ }^{[5]}$ as a new type of technology that converts environment mechanical energy into electrical energy. ${ }^{[3]}$ Compared to other technologies, TENG is advantageous in terms of high efficiency, high power density, light weight, low cost, and great manufacturability. ${ }^{[2,6-8]}$ TENG operates based on the coupling effects of contact electrification and electrostatic induction. The working principle requires two dissimilar surfaces to be oppositely charged upon contact. The common positive materials in TENG are polyamides, ${ }^{[9]}$ metals, ${ }^{[2]}$ indium tin oxide (ITO), ${ }^{[3]}$ and zinc oxide, ${ }^{[10]}$ and negative materials include fluorinated ethylene propylene $(\mathrm{FEP}),{ }^{[1,12]}$ polytetrafluoroethylene (PTFE) ${ }^{[9]}$ and polyvinylidene fluoride (PVDF), ${ }^{[2]}$ polydimethylsiloxane (PDMS), ${ }^{[3,10,13,14]}$ and polyethylene terephthalate (PET). ${ }^{[8,13-15]}$ The positive and negative materials are typically used in pairs in TENGs due to their opposite tendencies to gain or lose charges upon contact.

Cellulose, the most abundant natural polymer on Earth, can also be a good candidate as a triboelectric material. Cellulose nanofibrils (CNF) thin films, which are assembled from nanosized cellulose fibrils, are highly transparent, ${ }^{[16-18]}$ biodegradable,${ }^{[18]}$ flexible, ${ }^{[19]}$ and possess desirable surface roughness and electrical properties. ${ }^{[18,20]}$ As a result, CNF thin films are increasingly explored in modern electronic and optoelectronic devices. ${ }^{[3,7,8,13,18]}$ Due to the inclusion of abundant oxygen atoms, natural cellulose exhibits high tendency of losing electrons and thus becomes positively charged. ${ }^{[21]}$ Therefore, it can be paired with a wide range of triboelectric negative materials, such as FEP, PTFE, and PET to assemble TENGs. However, 
although recent work has predicted the promise of using cellulose as an actively TENG material, ${ }^{[22]}$ no practical TENG development on cellulose has been reported so far. In addition, cellulose can also be chemically modified to further tune their electronegativity. This paper reports the implementation of CNF materials in TENG development. Flexible and transparent CNF thin films with nanostructured surface were paired with FEP (fluorinated ethylene propylene) and operated in the vertical contact-separation mode. The TENG device exhibited comparable performance to the reported TENG counterparts built on synthetic polymers. The CNF-based TENG was also integrated within fiberboard made from recycled cardboard, creating a power board with high electric outputs using environmental-friendly wood-extracted materials.

\section{Experimental section}

Fabrication of CNF film: First, CNF hydrogel was prepared from wood pulp by tetramethylpiperidine-1-oxy (TEMPO)-mediated oxidation and subsequent mechanical homogenization following the method from Saito et al. ${ }^{[23]}$ Commercially supplied, bleached kraft eucalyptus pulps were oxidized in a mixture of TEMPO, sodium hypochlorite $(\mathrm{NaClO})$, and sodium chlorite $\left(\mathrm{NaClO}_{2}\right)$ under $\mathrm{pH}$ 6.8. The oxidation was carried out at $60{ }^{\circ} \mathrm{C}$ for $48 \mathrm{~h}$. Oxidized pulps were thoroughly washed in distilled water and refined in a disk refiner to break apart the residual fiber bundles. The refined fibers were then separated by centrifuge to remove the supernatant fraction, and concentrated to $1 \mathrm{wt} \%$ using ultrafiltration. Finally, this suspension was subjected to high pressure mechanical homogenization by passing through a series of 200and $87-\mu \mathrm{m}$ chambers on a microfluidizer for three times (M-110EH-30 Microfluidizer, Microfluidics, Newton, MA, USA). The resulting mixture of nanofibrils and water formed a transparent, stable aqueous colloid system (with a cellulose solid weight of $1 \%$ ), i.e., CNF 
hydrogel. To obtain the flexible and transparent CNF film, the as-processed CNF hydrogel was diluted with DI water and then filtered under approximately 0.55 MPa air pressure in a filtration system (Millipore Corporation, USA). In the filtration chamber, water within the slurry passed through a polytetrafluoroethylene membrane $(0.1 \mu \mathrm{m}$ pore sizes), leaving the CNF filter slab. After separation from the membrane, the CNF slab was sandwiched between layers of waxy coated paper, filter paper, and caul plates for room temperature drying followed by $65^{\circ} \mathrm{C}$ oven drying. During drying, pressure was applied on top by weights to prevent warping and wrinkling. Consequently, we obtained the flat, transparent and flexible CNF film.

Assembly of CNF film TENGs: The TENG used in demonstrating the design and performance of CNF-based TENGs was fabricated as follows: a pure CNF film with a size of $1 \mathrm{~cm} \times 1 \mathrm{~cm}$ was attached to the center of an ITO/PET substrate $(2 \mathrm{~cm} \times 5 \mathrm{~cm})$, which was considered as the top electrode. Bottom electrode comprised fluorinated ethylene propylene (FEP) with the same size and location on the other ITO/PET substrate. The two electrodes were separated by $1 \mathrm{~mm}$ spacers, and connected to the external circuit through copper tapes. Thickness of the CNF was $320 \mu \mathrm{m}$; while thickness of the FEP was $60 \mu \mathrm{m}$. TENG devices with various active areas from 1 to $40 \mathrm{~cm}^{2}$ were used in the performance characterization.

Manufacturing of power fiberboard: Fibers recovered from cardboard were dispersed in water and formed a uniform mixture by mechanical stirring. The mixture was forced through a screen by vacuum sucking, leaving a wet mat. An $8 \mathrm{~cm} \times 8 \mathrm{~cm}$ CNF-based TENG with all peripheries sealed was embedded in the wet mat during vacuum sucking. Subsequently, the mat was sandwiched between release papers and coil plates, and sent to cold pressing under a pressure of $100 \mathrm{MPa}$ for $1 \mathrm{~h}$. The obtained mat was then dried for $24 \mathrm{~h}$ at $65{ }^{\circ} \mathrm{C}$ under pressing by a $50 \mathrm{lb}$ weight. The diameter of the power fiberboard was $\sim 20 \mathrm{~cm}$, and the thickness was $\sim 4.5 \mathrm{~mm}$. 
Characterizations and measurements: A LEO 1530 Gemini field emission SEM and a Park AFM XE70 system were used to characterize the morphology and surface features of the CNF films. Performance of the CNF-based TENGs was characterized by applying periodical force. The top electrode was pressed down by a computer controlled shaker at a frequency of 10 to 60 $\mathrm{Hz}$, while the bottom electrode was anchored on a lab bench. Performance of the triboelectric fiberboard was measured when a person of normal weight stepped on and off it. The voltage output was measured using an Agilent DSO1012A oscilloscope. The input impedance of the oscilloscope is $1 \mathrm{M} \Omega$. Current output and charge transfer were measured by an Autolab PGSTAT302N station.

\section{Results and discussion}

The fabrication of CNF film started with manufacturing of CNF hydrogel, a process where wood pulp was oxidized and then mechanically homogenized according to Saito's method ${ }^{[23]}$ to achieve water dispersion of cellulose nanofibrils. The CNF hydrogel was then filtered and dried under pressure to obtain transparent and flexible CNF thin films (detailed fabrication procedures are included in the method section). Thickness of the CNF films was controlled in the range of 70 320 $\mu \mathrm{m}$ by varying the amount of CNF hydrogel. The as-prepared CNF films exhibited a fairly flat surface without any observable pin holes under low-magnification scanning electron microscope (Figure S1a). The fibrous feature could be clearly observed by AFM topography scan. As shown in Figure 1a, a typical fiber had a diameter of 230nm. The maximum height difference was approximately $300 \mathrm{~nm}$, suggesting the surface roughness was in $\sim 300 \mathrm{~nm}$. The asprepared film also showed excellent transparency and flexibility (inset of Figure 1b). The film can be bent a great degree and returned to its original shape after releasing without showing any 
marks. Chemical structure and FTIR spectrum of the CNF film is shown in Figure S1b, where the peak at $1050 \mathrm{~cm}^{-1}$ is assigned to the C-O-C stretching within the pyranose ring skeletal, and the $3500 \mathrm{~cm}^{-1}$ band is assigned to the $\mathrm{O}-\mathrm{H}$ stretching. The strong covalent bonding within each cellulose polymer chain and the hydrogen bonding between the polymer chains gave rise to a good stability of the CNF films in air, thus made CNF a good active material for TENGs to produce long-term steady output.

The CNF film was used as one active layer and pair with an FEP film to assemble a TENG. FEP was chosen due to its very negative position on the triboelectric series (i.e. far from cellulose). The TENG device was built with an identical size $(1 \mathrm{~cm} \times 1 \mathrm{~cm})$ of both CNF and FEP films on flexible ITO/PET substrate. The distance between the two ITO electrodes was fixed to be $1 \mathrm{~mm}$ for all experiments in this study. Schematic design and digital photo of the device are shown in Figure 1b, where the digital photo shows good transparency of the TENG device. Triboelectric performance of this CNF-based TENG was studied by periodically pressing/releasing the top surface under a constant force and frequency. Figures 1c show typical open-circuit voltage $\left(V_{o c}\right)$ measured from this TENG. Corresponding short circuit current $\left(I_{s c}\right)$ output signals are included in the supporting information (Figure S1c). The average peak values of the $V_{o c}$ and $I_{s c}$ reached $\sim 5 \mathrm{~V}$ and $\sim 5 \mu \mathrm{A}$, respectively. These values are comparable with the performance of TENGs made of typical synthetic polymer pairs such as Kapton-PET ${ }^{[5]}$ and PTFE-polyamide. ${ }^{[9]}$ It is noticed that the output voltage and current peaks were not symmetric. This is due to the fact that during each press-and-release cycle, the potential equilibrium state of the two surfaces may not always correspond to the point when the two films are farthest apart. To test the material and device stability, long-term operation was applied to the CNF-based 
TENG and steady voltage outputs were obtained after $10,5 \times 10^{3}, 2 \times 10^{4}, 1 \times 10^{5}$, and $5 \times 10^{5}$ cycles of $10 \mathrm{~Hz}$ mechanical impact (Figure S1d).

An interesting surface area-related $V_{o c}$ variation was observed when TENGs were made and tested from CNF/FEP films of five different surface areas in the range from $1 \mathrm{~cm}^{2}$ to $40 \mathrm{~cm}^{2}$. In this case, all TENGs had identical electrode spacing of $1 \mathrm{~mm}$. The $V_{o c}$ profiles measured from each TENG are shown together in Figure 1d and corresponding $I_{s c}$ profiles are included in Figure S2a. The measurement revealed that both $V_{o c}$ and $I_{s c}$ increased monotonically with the increase of active area. The maximum $V_{o c}$ and $I_{s c}$ were $32.8 \mathrm{~V}$ and $35 \mu \mathrm{A}$, corresponding to $40 \mathrm{~cm}^{2}$ surface area. To better understand the surface area-related $V_{o c}$ variation, the average values of the peak $V_{o c}$ were extracted and plotted as a function of the active surface area (solid circles in Figure 2a, the peak $I_{s c}$ V.s. active surface area was given in Figure S2b). The principle of TENG describes that the surface charge $\left(Q_{0}\right)$ is directly proportional to effective surface area $\left(A_{e f f}\right)$. This principle was proved by the linear relationship identified between the charge flux and $A_{\text {eff }}$ (pink diamonds in Figure 2a, corresponding charge flux profiles are included in Figure S2c). Therefore, the ratio between $Q_{0}$ and $A_{\text {eff }}$ remained constant for the TENG. This relationship tells that $V_{o c}$ should be a constant as a function of $A_{\text {eff }}$ following the equation $V=\frac{Q_{0}}{C_{0}}=\frac{Q_{0} d}{A_{\text {eff }} \varepsilon}$. By examining the testing protocols, we hypothesize that voltage-surface area relation was likely a result of the discharging behavior of TENGs in a resistor-capacitor (RC) circuit, where the resistance of the oscilloscope is not infinitely large. Since $\mathrm{C}$ is directly related to $A_{\text {eff, }}$ larger area TENG has larger time constant $\tau=R C$. Therefore, given all TENGs have the same initial $V_{o c}$, their $V \sim t$ relationships were calculated and plotted (detailed calculation is included in the Supporting Information S2). As shown in Figure 2b, TENG with larger $A_{\text {eff }}$ demonstrates slower decaying curve. Considering the finite loading resistance $(\sim 1 \mathrm{M} \Omega)$ and data sampling rate $\left(1.6 \times 10^{-5} \mathrm{~s}\right)$ of the oscilloscope, 
although initial $V_{o c}$ might be the same for all TENGs, $A_{\text {eff }}$-related voltage values could be recorded at any time $t(t>0)$. The blue circles in Figure 2a represent the calculated voltage values obtained at $t=1.6 \times 10^{-5} \mathrm{~s}$ (indicated by the red dashed line in Figure $2 \mathrm{~b}$ ), which demonstrated excellent matching trend as the experimental values. To further confirm this charge releasing hypothesis, the output voltages were further measured when the sampling rate of the oscilloscope was tuned to be $5.86 \times 10^{-5} \mathrm{~s}$. As shown in Figure S2d, the peak $V_{\text {oc }}$ were found smaller than previous values and matched well with the calculated values. As the $40 \mathrm{~cm}^{2}$ film demonstrated high voltage output and $I_{s c}$, later experiments in this study all used films of $40 \mathrm{~cm}^{2}$ surface areas unless otherwise specified.

External load matching for the CNF TENG was carried out to further characterize its output performance. Figure 2c shows the dependence of the peak $V_{o c}$ and $I_{s c}$ as a function of external load resistance measured from a TENG with a CNF film of $70 \mu \mathrm{m}$ thickness and $40 \mathrm{~cm}^{2}$ surface areas. Same as other TENGs, the $V_{o c}$ monotonically increased with the rising of load resistance while $I_{s c}$ showed an opposite trend as a result of Ohmic loss. Corresponding power output was calculated from the product of $V_{o c}$ and $I_{s c}$ and was plotted in Figure $2 \mathrm{~d}$. The optimal power point was obtained at $\sim 1 \mathrm{M} \Omega$ with a peak value of $0.56 \mathrm{~mW}$. It should be noted that the power value presented here is the instantaneous peak power, corresponding to the short and sharp voltage and current peaks (Figure S3a and b). Analyzing the amount of electrical energy produced would be a more practical approach to evaluate its applicant potential.

In this regard, the CNF TENG was used to charge capacitors, so as to explore its potential as a continuous direct current (DC) energy supply. The experiments were conducted with the TENG connected to the capacitor through a rectification circuit, which converted the alternative current (AC)-type electric output into one direction (Figure S3c and d). When the TENG was under 
continuous impacts with different frequencies, the voltage across the capacitor was measured every 5 seconds for a total of 110 seconds. Typical voltage raising profiles across a $10 \mu \mathrm{F}$ capacitor is shown in Figure 3a. A saturation charging curve was obtained after $\sim 40$ s operation, which is typical for resistor-capacitor (RC) charging. ${ }^{[2]}$ The charging of larger capacitors, e.g. $33 \mu \mathrm{F}$ and $68 \mu \mathrm{F}$ capacitor, exhibited similar behavior, while larger capacitor required longer time to reach the saturation voltage (Figure S4a and S4b). Further observation revealed that a higher frequency gave rise to a higher saturation voltage. It was because the equilibrium was established between the TENG's charging rate and the capacitor's leakage rate. ${ }^{[25]}$ Based on the charging curves, the energy (E) stored in the capacitor were calculated following the equations: $E=C U^{2} / 2$, where $C$ is the capacitance, $U$ is the saturation voltage. The result of the calculations was plotted in Figure 3b. With the increase of frequency within the studied range, the output energy raised monotonically from 0.01 to $0.16 \mathrm{~mJ}$. Very little variation of energy output was observed among the three different capacitors. Since the measurements showed the minimum output occurred at the lowest frequency $(10 \mathrm{~Hz})$, we tested the feasibility of the TENG as a power source for LEDs at this lowest frequency. As shown in the inset of Figure 3b, the TENG was able to light up 150 green LEDs that were connected in series, proving its capability as a practical DC power source.

Considering that one major application of wood-extracted fibers is all kinds of board, our successful development of TENG using CNF film allows the creation of powering boards that are made from the same green and biodegradable nature materials. To demonstrate this concept, CNF-based TENG was integrated with a fiberboard made from recycled cardboards. Figure 4a showed the manufacturing process of the triboelectric fiberboard. An $8 \mathrm{~cm} \times 8 \mathrm{~cm}$ CNF TENG with all peripheries sealed was embedded in the wet fibers that were recovered from cardboard. 
These raw fibers were then formed into fibermat by vacuum sucking and further hardened by cold pressing at $100 \mathrm{MPa}$ for 1 hour. After drying at $65^{\circ} \mathrm{C}$ under $50 \mathrm{lb}$ weight for $24 \mathrm{~h}$, a fiberboard was obtained with a typical dimension of $20 \mathrm{~cm}$ in diameter and $4 \mathrm{~mm}$ in thickness. To demonstrate the powering capability of this TENG-integrated fiberboard, a person of normal weight repeatedly stepped on the fiberboard and corresponding electric output was monitored. As shown in Figure $4 \mathrm{~b}$ and $\mathrm{c}$, the peak $V_{o c}$ and $I_{s c}$ were ranged between $\sim 10-30 \mathrm{~V}$ and $\sim 10-90$ $\mu \mathrm{A}$, respectively, due to the irregular stepping force and speed. More practically, one single step on this fiberboard can easily light up 35 green LEDs connected in series (panel 6 in Figure 4a and supporting video S1), demonstrating the promise of powering commercial electronics. By solving charge output based on a triple-capacitor model (Figure S5), we found that the power board could output up to $98 \%$ triboelectric charge to the external circuit, revealing a very high internal efficiency of charge output. The conversion efficiency from input mechanical energy to output electrical energy was calculated to be $\sim 8.3 \%$. The relatively low energy conversion efficiency might because that the input mechanical energy is mostly stored as mechanical deformation due to the rigidity of the fiberboard itself.

\section{Conclusion}

In summary, CNF thin films were successfully used in TENG development. Using renewable, biodegradable, and abundant natural CNFs for TENG application holds great potential for a new type of ecofriendly mechanical energy harvesting system. The electrical output of the CNF-FEP TENG is comparable to the typical synthetic polymer TENG devices. The measured triboelectric voltage output was found to be a function of the CNF film size, which was attributed to timedependent triboelectric discharging behavior. Through regular capacitor charging, the output 
electric energy was found in the range of 0.01 to $0.16 \mathrm{~mJ}$ as a function of the mechanical impact frequency. CNF-based TENG was further integrated with a fiberboard fabricated from recycled cardboard fibers using a chemical-free cold pressing method. This is a very simple, ecofriendly and cost-effective manufacturing process. This fiberboard has excellent integrity upon mechanical impacts as well as great recyclability. It produced up to $\sim 30 \mathrm{~V}$ and $\sim 90 \mu \mathrm{A}$ electric outputs when it subjected to a normal human step, which lighted up 35 green LEDs assembled together with the power fiberboard. Calculations showed that the power fiberboard has a charge transfer efficiency of up to $\sim 98 \%$. This development showed a great promise in creating largescale and environmentally sustainable triboelectric wood/fiber boards or floorings. Combining the unique triboelectric mechanical energy harvesting capability and ecofriendliness of CNFs and wooden fibers, and the wide modification possibilities of CNFs, it is foreseeable that CNFs and other natural wood-extracted materials will play an important role in developing and manufacturing industrial-level flooring, packaging and supporting infrastructures with such a unique capability of effectively harvesting mechanical energy from the ambient environment.

\section{Acknowledgements}

C.Y. and A.H. contribute equally to this work. The authors thank financial support from the Forrest Product Laboratory under Award \# 14-JV-11111124-029 and National Science Foundation (NSF) under award \# CMMI-1148919. C.Y. and A.H. thank Matthew Starr and Zhaodong Li for helpful discussions, thank Dalong Geng for AFM scanning, and John Hunt for the help with fiberboard manufacturing. 


\section{References}

[1] J. Zhong, Q. Zhong, F. Fan, Y. Zhang, S. Wang, B. Hu, Z. L. Wang, J. Zhou, Nano Energy 2 (2013) 491.

[2] G. Zhu, Y. S. Zhou, P. Bai, X. S. Meng, Q. Jing, J. Chen, Z. L. Wang, Adv. Mater. 26 (2014) 3788 .

[3] F. R. Fan, J. Luo, W. Tang, C. Li, C. Zhang, Z. Tian, Z. L. Wang, J. Mater. Chem. A 2 (2014) 13219.

[4] Y. Xie, S. Wang, L. Lin, Q. Jing, Z.-H. Lin, S. Niu, Z. Wu, Z. L. Wang, ACS Nano 7 (2013) 7119.

[5] F.-R. Fan, Z.-Q. Tian, Z. Lin Wang, Nano Energy 1 (2012) 328.

[6] G. Zhu, J. Chen, T. Zhang, Q. Jing, Z. L. Wang, Nat. Commun. 5 (2014) 3426.

[7] Q. Liang, X. Yan, Y. Gu, K. Zhang, M. Liang, S. Lu, X. Zheng, Y. Zhang, Sci. Rep. 5 (2015) 9080.

[8] K. Y. Lee, M. K. Gupta, S.-W. Kim, Nano Energy 14 (2014) 139.

[9] Y. Yang, H. Zhang, R. Liu, X. Wen, T. C. Hou, Z. L. Wang, Adv. Energy Mater. 3 (2013) 1563.

[10] Y. H. Ko, G. Nagaraju, S. H. Lee, J. S. Yu, ACS Appl. Mater. Interfaces 6 (2014) 6631.

[11] L. Lin, Y. Xie, S. Niu, S. Wang, P.-K. Yang, Z. L. Wang, ACS Nano 9 (2015) 922

[12] Y. Yang, G. Zhu, H. Zhang, J. Chen, X. Zhong, Z.-H. Lin, Y. Su, P. Bai, X. Wen, Z. L. Wang, ACS Nano 7 (2013) 9461 
[13] F.-R. Fan, L. Lin, G. Zhu, W. Wu, R. Zhang, Z. L. Wang, Nano Lett 12 (2012) 3109.

[14] B. Meng, W. Tang, Z.-h. Too, X. Zhang, M. Han, W. Liu, H. Zhang, Energy Environ. Sci. 6 (2013) 3235.

[15] L. M. Zhang, F. Xue, W. M. Du, C. B. Han, C. Zhang, Z. L. Wang, Nano Res. 7 (2014) 1215.

[16] Z. Q. Fang, H. L. Zhu, Y. B. Yuan, D. Ha, S. Z. Zhu, C. Preston, Q. X. Chen, Y. Y. Li, X. G. Han, S. Lee, G. Chen, T. Li, J. Munday, J. S. Huang, L. B. Hu, Nano Lett. 14 (2014) 765

[17] M. Nogi, S. Iwamoto, A. N. Nakagaito, H. Yano, Adv. Mater. 21 (2009) 1595.

[18] Y. H. Jung, T.-H. Chang, H. Zhang, C. Yao, Q. Zheng, V. W. Yang, H. Mi, M. Kim, S. J. Cho, D.-W. Park, H. Jiang, J. Lee, Y. Qiu, W. Zhou, Z. Cai, S. Gong, Z. Ma, Nat. Commun. 6 (2015) 7170.

[19] H. Zhu, Z. Xiao, D. Liu, Y. Li, N. J. Weadock, Z. Fang, J. Huang, L. Hu, Energy Environ. Sci. 6 (2013) 2105.

[20] H. Zhu, Y. Li, Z. Fang, J. Xu, F. Cao, J. Wan, C. Preston, B. Yang, L. Hu, ACS Nano 8 (2014) 3606.

[21] A. Diaz, R. Felix-Navarro, J. Electrostat. 62 (2004) 277.

[22] Y. Zi, S. Niu, J. Wang, Z. Wen, W. Tang, ZL. Wang, Nat. Commun. 6 (2015) 8376.

[23] T. Saito, M. Hirota, N. Tamura, S. Kimura, H. Fukuzumi, L. Heux, A. Isogai, Biomacromolecules 10 (2009) 1992.

[24] S. Niu, Y. Liu, Y. S. Zhou, S. Wang, L. Lin, Z. L. Wang, IEEE Trans. Electron Dev. 62, (2015) 641.

[25] Y. Mao, P. Zhao, G. McConohy, H. Yang, Y. Tong, X. Wang, Adv. Energy Mater. 4 (2014) 1301624. 
Figures and Captions
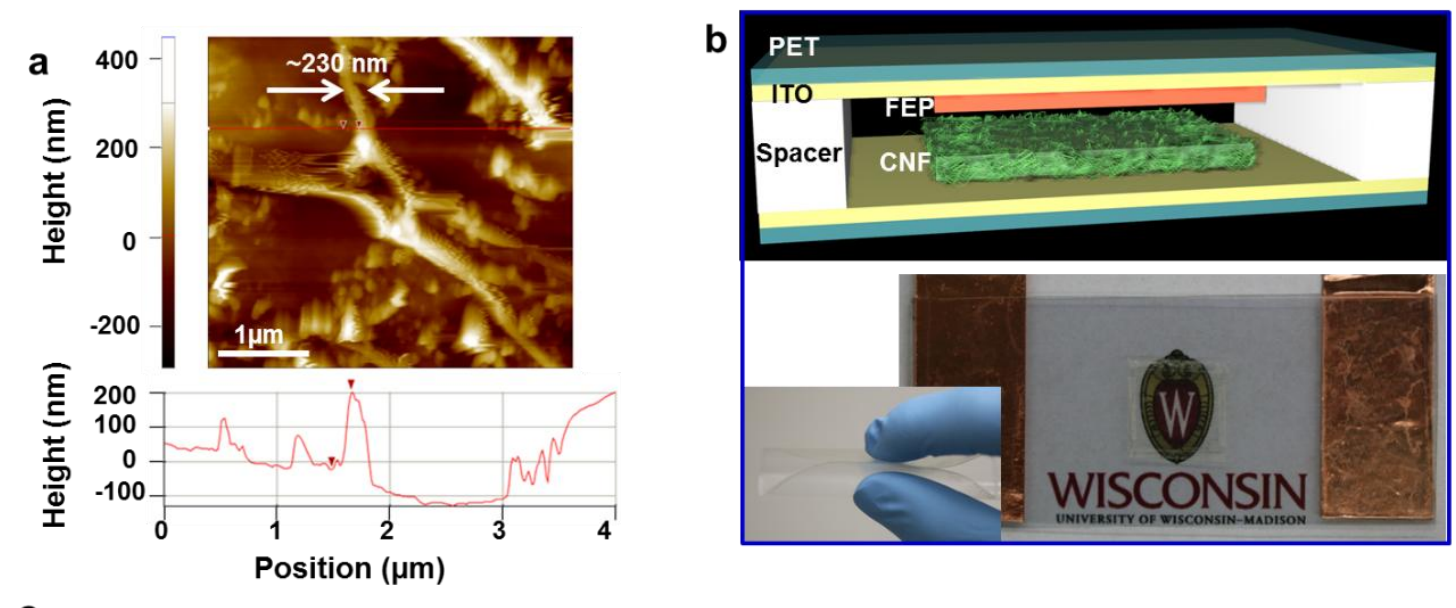

C

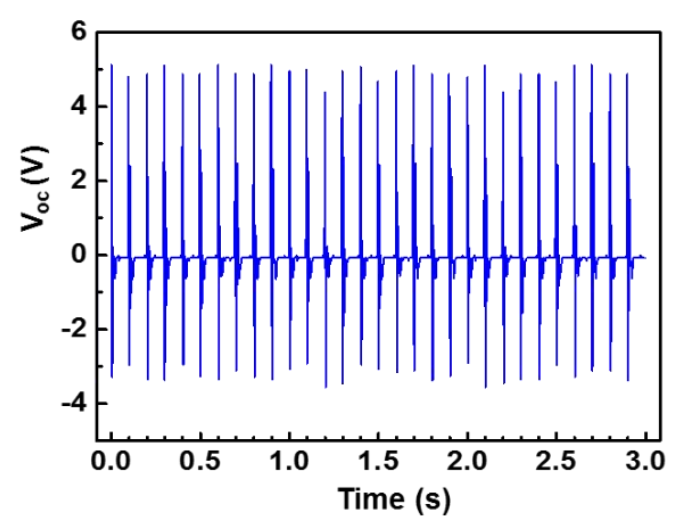

d

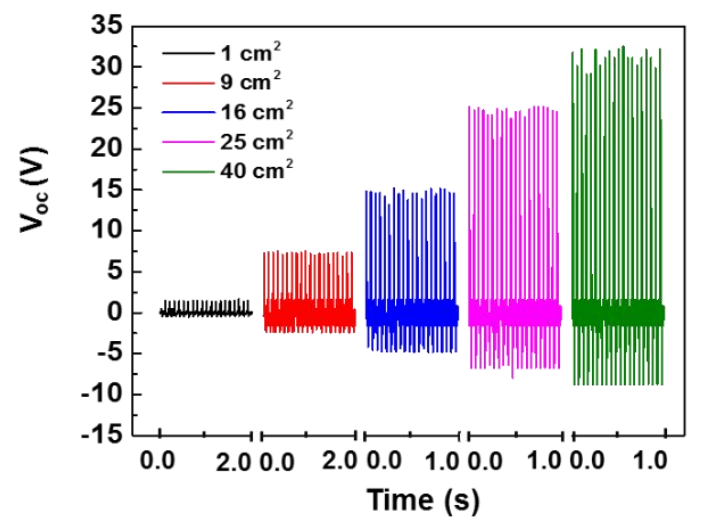

Figure 1. Design and performance of CNF film TENG. (a) AFM topography image of a CNF film surface. (b) Schematic drawing (top) and a digital photo (bottom) of the CNF film-based TENG, inset shows the flexibility of CNF film. (c) Open circuit voltage $\left(V_{o c}\right)$ from the CNF filmbased TENG. (d) $V_{o c}$ measured from CNF-based TENG with various active surface areas. 

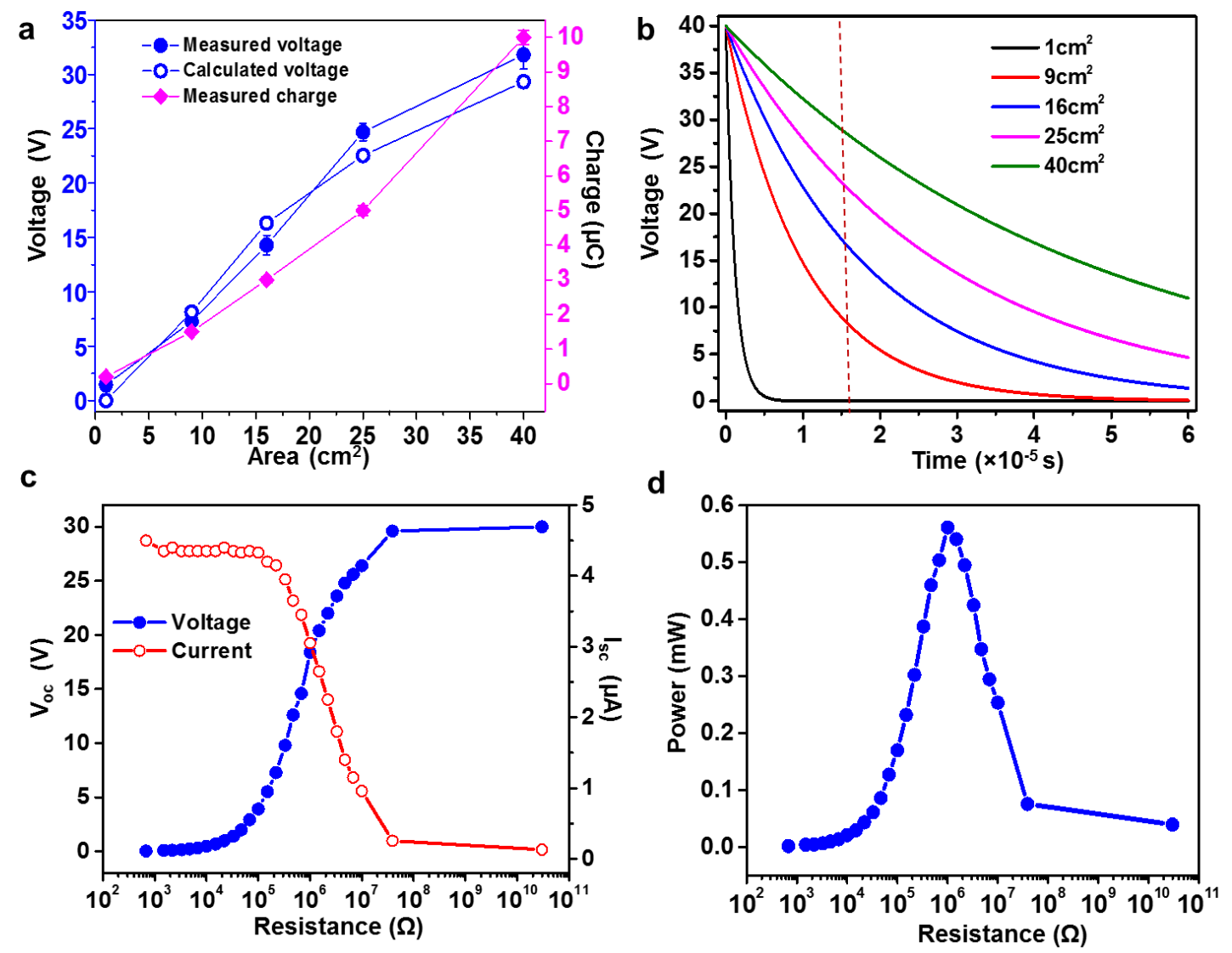

Figure 2. Output performance of the CNF film-based TENG. (a) Measured voltage output (blue dots), calculated voltage output (blue circles), and measured triboelectric charge transfer (purple diamonds) as a function of active CNF film area $\left(A_{\text {eff }}\right)$. (b) Calculated RC discharging curves for TENGs with different $A_{\text {eff. }}$ (c) Voltage and current output as a function of the load resistance. (d) Calculated output power as a function of the load resistance. 

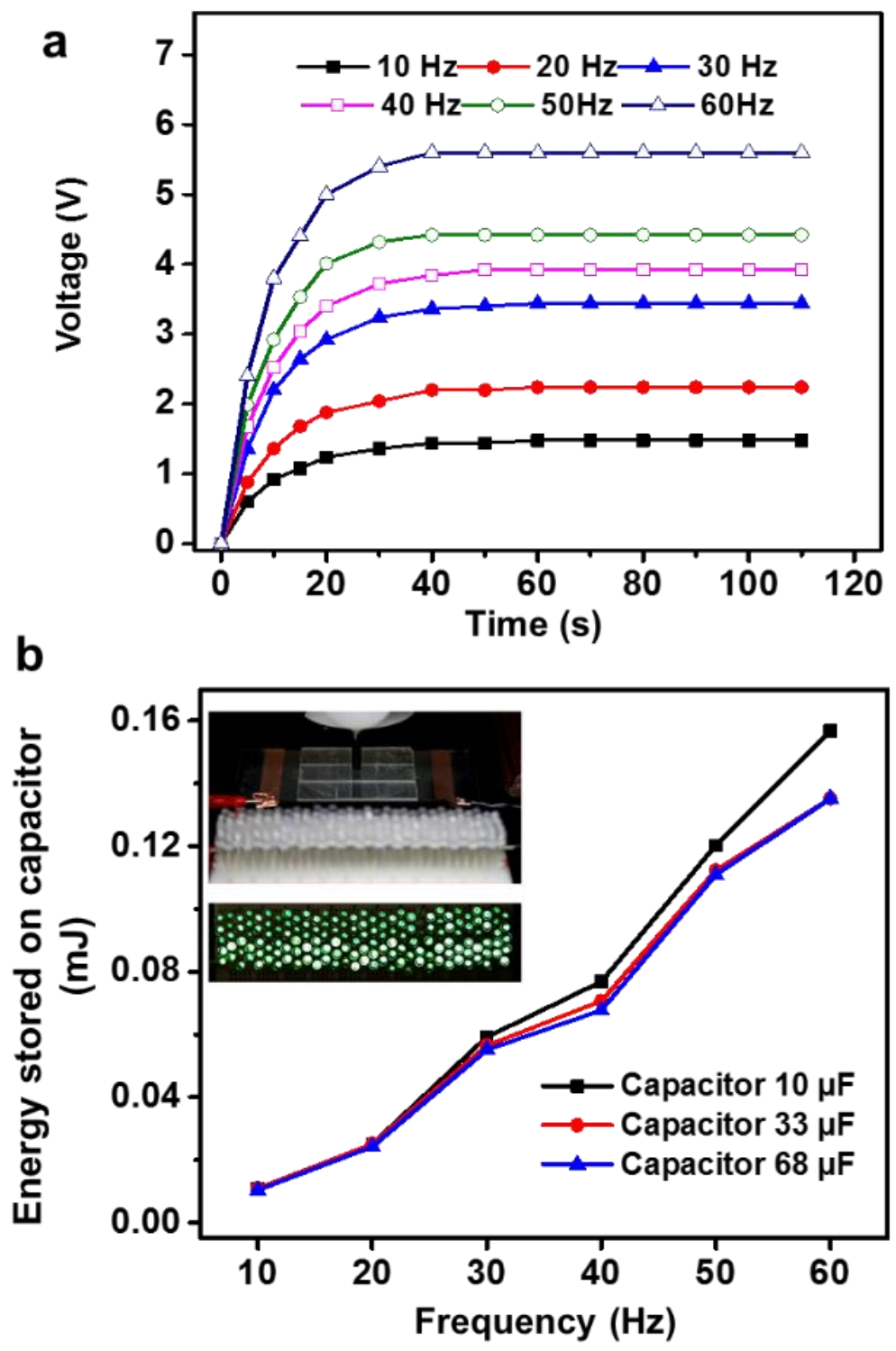

Figure 3. Capacitor charging performance of the TENG and demonstration of it as a direct power source. (a) Voltage measured across a $10 \mu \mathrm{F}$ capacitor when it was charged by the TENG under different frequencies of mechanical impacts. (b) The energy stored in capacitor calculated from the capacitor charging curves as a function of mechanical impact frequency. Inset shows the TENG is successfully used as a direct current power source to light up 150 green LEDs connected in series. 
a

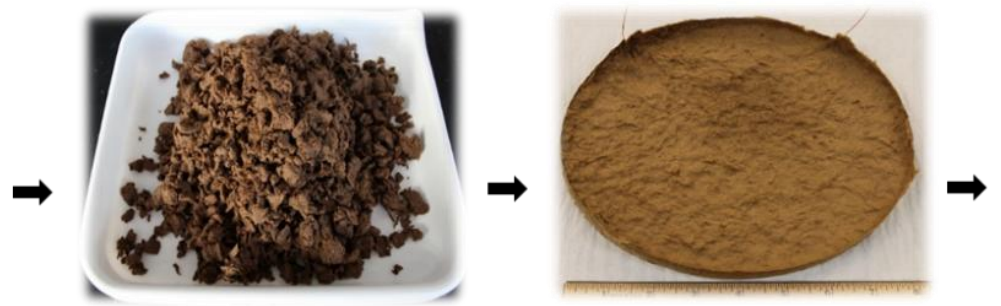

(1) CNF film TENG

(2) Recycled fibers from paper cards

(3) Fiber mat with the triboelectric unit embedded in
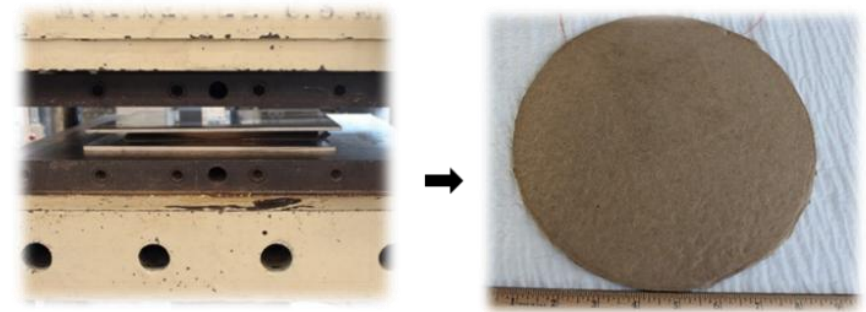

(4) Fiber mat under cold pressing

(5) Triboelectric fiberboard
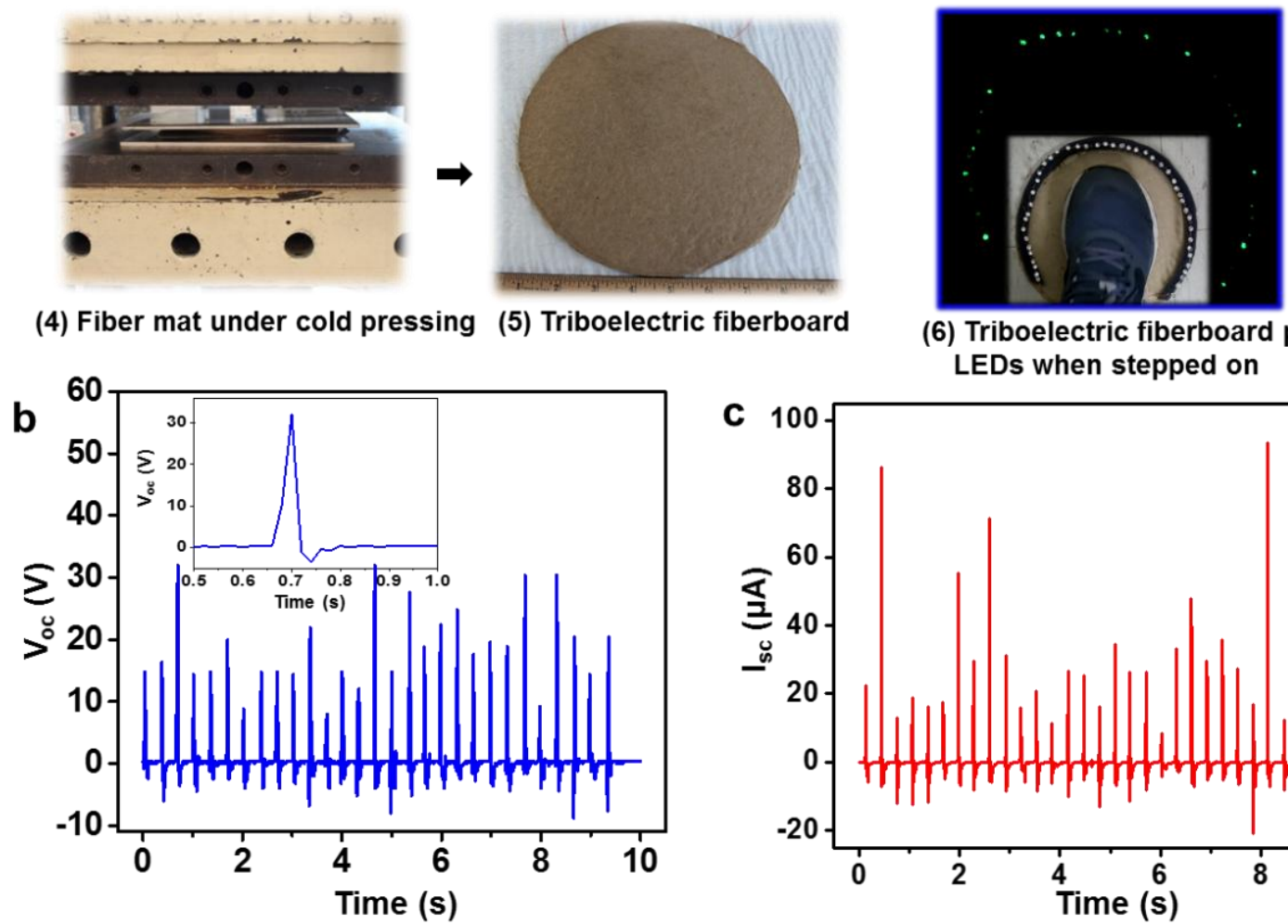

(6) Triboelectric fiberboard powering LEDs when stepped on

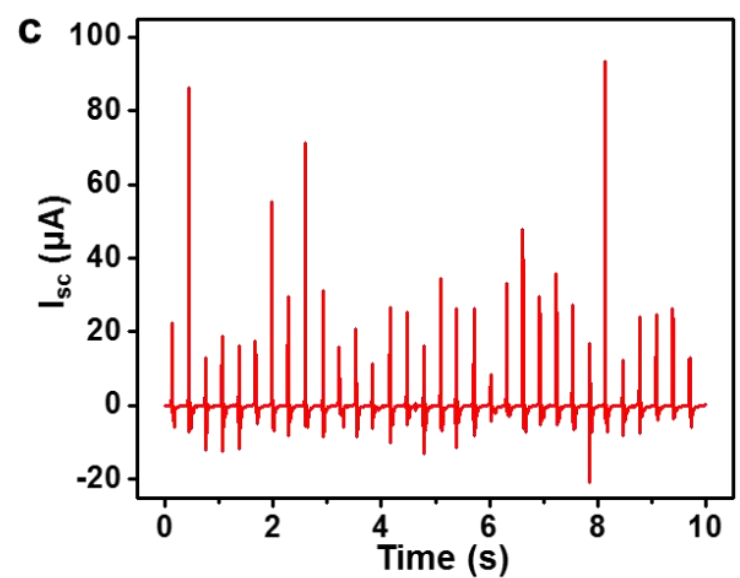

Figure 4. Manufacturing and performance of CNF-based TENG fiberboard. (a) Manufacturing process of the triboelectric fiberboard. (b) open-circuit voltage $\left(V_{o c}\right)$ and (c) short-circuit current $\left(I_{s c}\right)$ output of the triboelectric fiberboard when a person of normal weight stepped on repeatedly for $\sim 30$ times. Inset of (b) is the $V_{o c}$ during one stepping. 


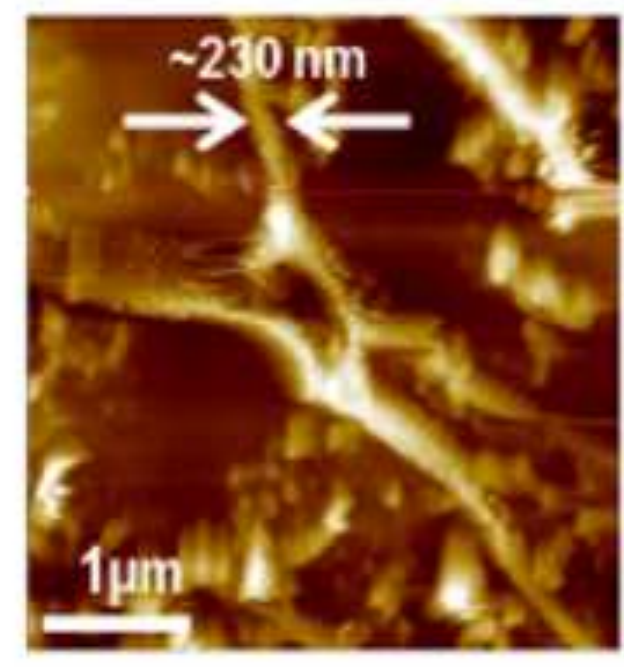

다믄
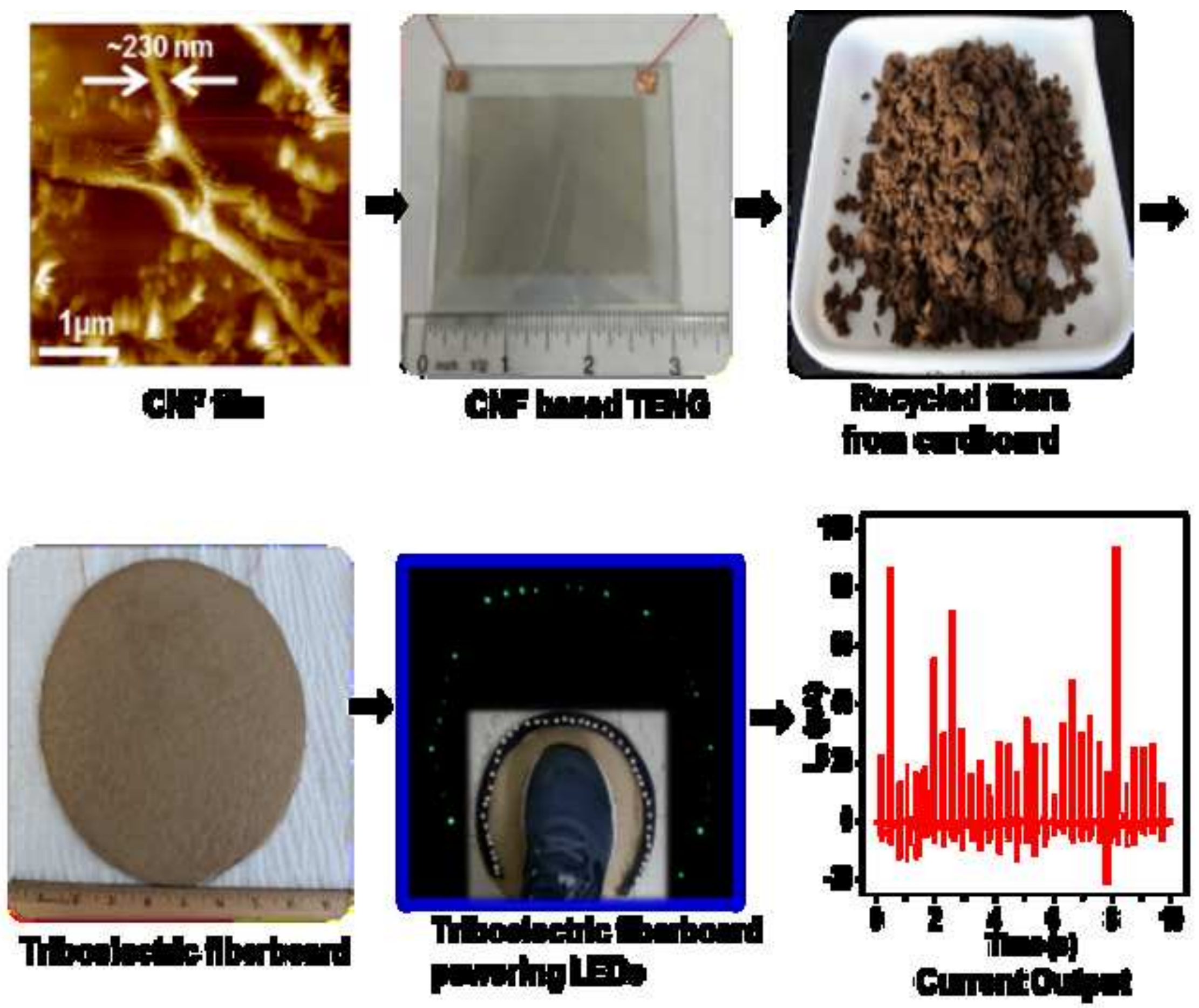